\title{
Conceptual design and performance simulations of super-compact electromagnetic calorimeter
}

\author{
Libor Skoda ${ }^{\mathrm{a}}$ \\ ENPP Group, FNSPE CTU in Prague, Czech Republic
}

\begin{abstract}
Measurements of particle production at forward rapidities in high energy p-p, p-A and A-A collisions provide access to physics processes at very low Bjorken $\mathrm{x}$. These measurements will allow to study the gluon saturation scale and improve our knowledge of parton distribution in nuclei. Specific requirements must be fulfilled for a calorimeter to successfully operate in high-multiplicity forward region within often stringent space limits. Here we present a study of a conceptual design of super-compact electromagnetic calorimeter being developed at Czech Technical University in Prague. The design of the sampling calorimeter is based on a sandwich structure of thin tungsten and scintillator layers oriented in parallel to the beam. Used optical readout of individual scintillator pads guaranties the required high radiation hardness of the detector. We present simulation of the expected performance of the optical pad readout together with overall detector performance. It is aimed for the detector to allow measuring of high energy photons $(1<\mathrm{E}<300 \mathrm{GeV})$ in forward rapidities $(2.5<\eta<4)$.
\end{abstract}

\section{Motivation}

Forward rapidity region of high energy particle collisions offers opportunity for studying more details of physics at small Bjorken-x. Particles produced to this region originate dominantly from gluon interactions providing possibility to scan low-x gluon densities. Basic phenomena allowing to study the physics aspects in this region are forward $\pi^{0}$ and jets production, prompt photon production, quarkonia and leptons from heavy quarks, etc. It will also improve our knowledge of parton distribution in nuclei.

A detector for this region must fulfil specific requirements - successfully operate in high multiplicity background, at high trigger rates, in strong radiation environment and electromagnetic fields and at limited space. The aim of our project is, therefore, to develop new super-compact electromagnetic calorimeter (ECAL) for the forward region based on the tungsten-scintillator calorimetry for wide pseudorapidity range for photon energies up to $\mathrm{E} \approx 300 \mathrm{GeV}[1]$.

\section{Design of the detector and simulations}

The mechanical design of the sampling calorimeter is based on a sandwich structure of thin tungsten and scintillator cards oriented in parallel to the beam (Figure 1). High purity tungsten plates (absorber) are $1.5 \mathrm{~mm}$ thick.

\footnotetext{
${ }^{a}$ Corresponding author: 1skoda@cern.ch
}

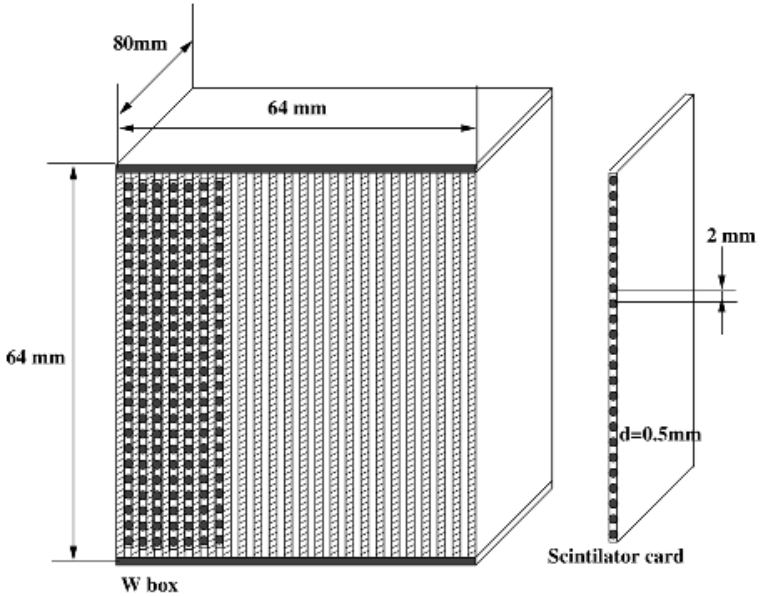

Figure 1. Calorimetric box with exchangeable scintillator cards.

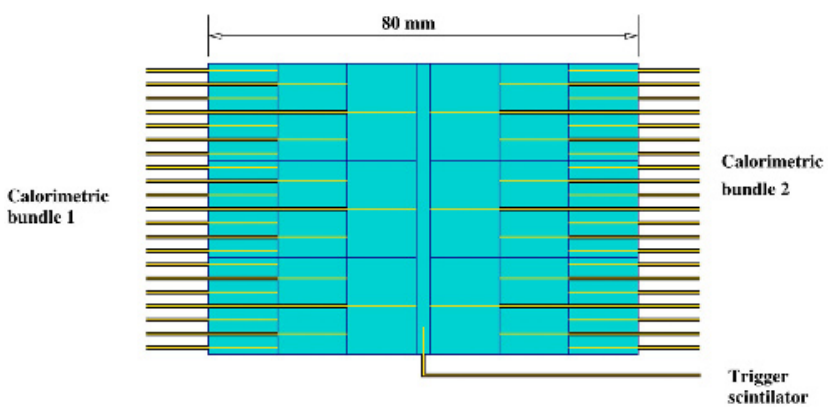

Figure 2. Scintillator card with optical fibers. 
Scintillator cards are $0.5 \mathrm{~mm}$ thick and consist of variable granularity pads from high quantum efficiency radiation hard inorganic scintillators (YAG, 3HF, ...). Due to variable granularity of the pad size the best resolution is in the first and the last layer while in the middle the signal is integrated at shower maximum. Triggering at shower maximum suppress noise and low energy particles (Figure 2).

Every pad is individually read out by radiation hard quartz optical low-attenuation fiber which lead signal from the scintillator to the image intensifier and the highspeed camera. Use of optical fibers allows to have signal processing outside the strong radiation environment. Thanks to camera properties (FastCamera13 - FastVision) it is possible to achieve single photon sensitivity and $5 \mathrm{kHz}$ full frame operation rate. Moreover, fast shutter gate $(<2$ ns) allows precise event selection (trigger) in time.

To improve readout from scintillator pads, the interconnection between pads and fibers is optimized - e.g. by spherical glass lens at the end of each optical fiber as it increases the detection area (Figure 3).

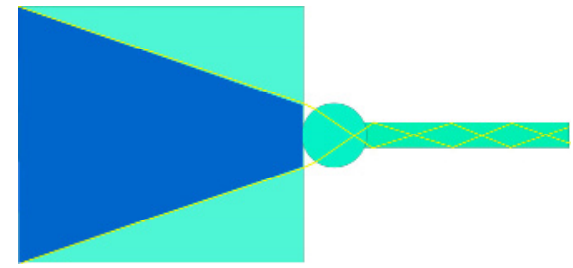

Figure 3. Glass lens at the end of optical fiber.

Moreover, energy resolution of the detector is examined as a function of generated particle energy using simulations from Geant 3. As one can see, the energy resolution for particle with energy higher than $20 \mathrm{GeV}$ is from $30 \%$ at $\eta=4$ to $50 \%$ at $\eta=2.5$ (Figure 4 ).

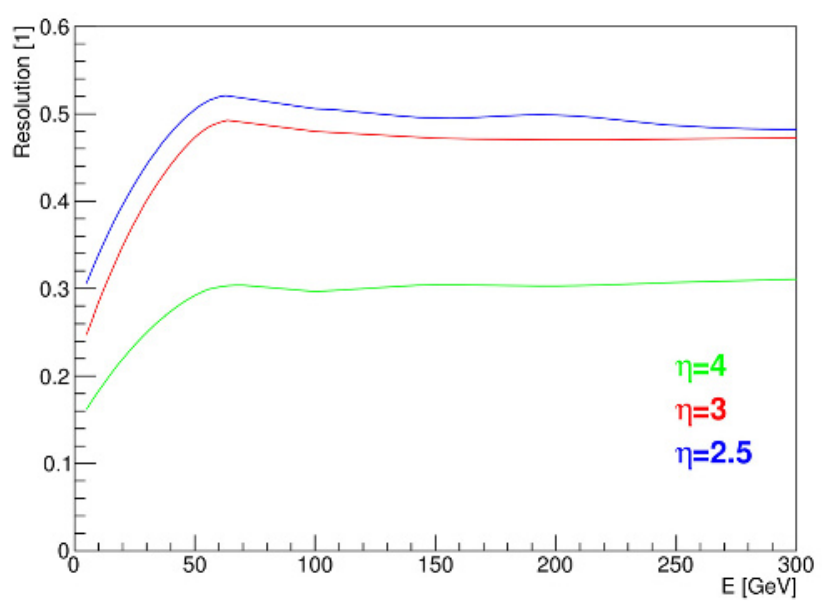

Figure 4. Energy resolution of the detector for different gamma energies.

Response of the detector to photons with energies ranging from 50 to $200 \mathrm{GeV}$ was examined and it is clearly visible, that reconstruction of the signal is possible even in the high multiplicity environment due to deposition of energy in each layer at different depths (Figure 5).
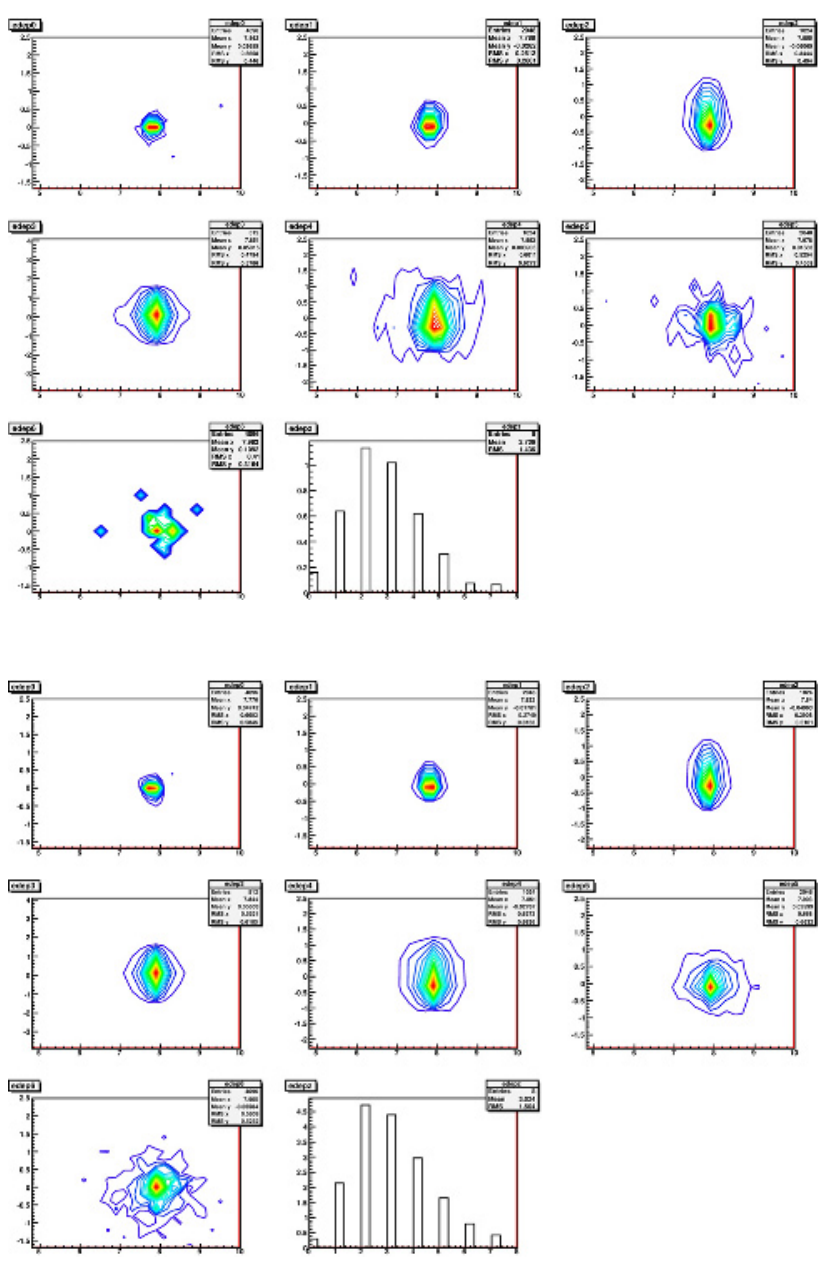

Figure 5. Response of the detector to particles at various energies - to $50 \mathrm{GeV}$ (up) and $200 \mathrm{GeV}$ (down).

\section{Summary}

Concept of super-compact ECAL for forward rapidity region has been examined in this paper allowing for $\gamma$ and $\pi^{0}$ identification up to energy $300 \mathrm{GeV}$ with full energy reconstruction. Calorimeter allows operation in high multiplicity environment close to $\eta=4$ with response to individual particles. Optical readout can operate at $\sim 5 \mathrm{kHz}$ trigger rate. Compact design and need of only thin support assure almost no dead zones. Design and used materials guarantee the required high radiation hardness of the detector. Electromagnetic fields do not affect its operation. Moreover, there is no need for cooling and power the detector. The prototype is being made for cosmic rays test and beam test (CERN).

\section{References}

1. J. Cepila, V. Petracek, L. Skoda, M. Petran, J. Adam, R. Smakal, PHYSICS AT LHC 2011 Proceedings of the International Conference held in Perugia, Italy, (2011), SLAC eConf C1106061

This work was supported by RVO 68407700. 